OPEN

SUBJECT AREAS:

ELECTRICAL AND

ELECTRONIC

ENGINEERING

ELECTRONIC DEVICES

Received

11 September 2013

Accepted

13 May 2014

Published

4 June 2014

Correspondence and requests for materials should be addressed to Y.-F.C. (yfchen@phys. ntu.edu.tw)

\section{Electrically and Optically Readable Light Emitting Memories}

\author{
Che-Wei Chang ${ }^{1,3}$, Wei-Chun Tan' , Meng-Lin Lu' , Tai-Chun Pan ${ }^{4}$, Ying-Jay Yang ${ }^{3} \&$ Yang-Fang Chen ${ }^{1,2}$
}

'Department of Physics, National Taiwan University, No. 1, Sec. 4, Roosevelt Road, Taipei 106, Taiwan, Republic of China, ${ }^{2}$ Center for Emerging Material and Advanced Devices, National Taiwan University, No. 1, Sec. 4, Roosevelt Road, Taipei 106, Taiwan, Republic of China, ${ }^{3}$ Graduate Institute of Electronics Engineering, National Taiwan University, No. 1, Sec. 4, Roosevelt Road, Taipei 106, Taiwan, Republic of China, ${ }^{4}$ Institute of Optoelectronic Sciences, National Taiwan Ocean University, No.2, Pei-Ning Road, Keelung 224, Taiwan, Republic of China.

Electrochemical metallization memories based on redox-induced resistance switching have been considered as the next-generation electronic storage devices. However, the electronic signals suffer from the interconnect delay and the limited reading speed, which are the major obstacles for memory performance. To solve this problem, here we demonstrate the first attempt of light-emitting memory (LEM) that uses $\mathrm{SiO}_{2}$ as the resistive switching material in tandem with graphene-insulator-semiconductor (GIS) light-emitting diode (LED). By utilizing the excellent properties of graphene, such as high conductivity, high robustness and high transparency, our proposed LEM enables data communication via electronic and optical signals simultaneously. Both the bistable light-emission state and the resistance switching properties can be attributed to the conducting filament mechanism. Moreover, on the analysis of current-voltage characteristics, we further confirm that the electroluminescence signal originates from the carrier tunneling, which is quite different from the standard $\mathrm{p}-\mathrm{n}$ junction model. We stress here that the newly developed LEM device possesses a simple structure with mature fabrication processes, which integrates advantages of all composed materials and can be extended to many other material systems. It should be able to attract academic interest as well as stimulate industrial application.

N umerous progresses have been achieved in the last several decades on discrete semiconductor decices, such as memories and light emitting diodes. Nowadays, both the performance and reliability of these devices have already become sufficient for use in practical applications. Future work may involve the research of devices that possess wider functionality and compatibility in a variety of data storage and processing. Notably, the demand of high-speed inter-chip and intra-chip link has dictated the developments of communication. The conventional electronic devices gradually approach their limit due to the increasing difficulties in controlling the carriers at shrinking sizes ${ }^{1,2}$. Among several candidates for next-generation memory cells, resistive random access memory (RRAM) based on a simple two-terminal electrical switch has the potential to serve as a replacement for conventional memory structures due to its good switching properties, low power consumption and especially, three-dimensional multilayer stacking to achieve high density memory ${ }^{3-8}$. The typical currentvoltage (I-V) characteristics of the RRAM cells exhibit an extreme change in resistance between high resistance state (HRS) and low resistance state (LRS). One of the underlying mechanisms responsible for the RRAMs arises from the formation of conducting filament networks under the application of a positive voltage across the cell. Upon application of a negative bias, the conducting filaments are dissolved and the memory cell switches to the HRS. However, the reading process is still in a serial sequence, that is, the data is transmitted by scanning one bit after another. In application, there is much interest in developing the reading process in parallel, which will boost the data transmission rate dramatically. One of the promising ways in realizing paralleling reading process is the optical interconnect. For example, when the light-emitting memories (LEMs) are turned on, data are received by optical sensor (e.g., CCD), i.e., regions without light emission are served as logic "0", and regions with light emission are served as logic "1". By monitoring the whole LEM array, optical sensor can sense and distinguish each light emission generated by LEMs at the same time. Therefore, the maximum data throughput can be greatly increased, which depends on the density of LEM array and the capability of optical sensor. To achieve the above described functionality, the integration of light emitter and memory device is definitely one of the feasible approaches. In this work, we made the first attempt of an inorganic LEM, which enables data communication via optical and electrical detections and provides both parallel reading process and high data bandwidth simultaneously. 
The group III-nitride based semiconductors have been the focus of promising application in optoelectronics devices with its mature fabrication technologies and the composition dependent variable bandgap. The InGaN based light-emitting diodes (LEDs) and laser diodes (LDs) can cover the light spectrum from UV to visible regions ${ }^{9-15}$. Although the InGaN based technology is well developed, it requires complex process involving multiple-quantum-wells (MQWs). In this work, the LEM was achieved by developing the graphene-insulator-semiconductor (GIS) light-emitting diode (LED) in tandem with a $\mathrm{SiO}_{2}$ based memory cell. The GIS-LEDs do not rely on $\mathrm{p}-\mathrm{n}$ junctions, which enables to expand the list of usable semiconductors to materials that cannot be easily doped and can be economically fabricated without difficulties. We constructed the GIS-LEDs by using graphene layer as a metal contact on $\mathrm{p}-\mathrm{GaN}$ substrate with an insulating $\mathrm{SiO}_{2}$ interlayer.

Among the group of III-nitride based semiconductors, GaN serves as the pillar of its family. Compared with the ternary InGaN alloys, the growth of good quality of GaN film is relatively easy, which can produce a strong light emission. Moreover, the combination of blue light with phosphor powders or quantum dots with suitable concentrations can generate the desired color of light. Based on this advantage, the device with blue-light illumination enables controllable color generation. In addition, the choice of p-type $\mathrm{GaN}$ film is because the barrier height of $\mathrm{SiO}_{2} / \mathrm{n}-\mathrm{GaN}$ device is much lower than that of $\mathrm{SiO}_{2} /$ $\mathrm{p}-\mathrm{GaN}$. Therefore, one of the benefits for using $\mathrm{p}-\mathrm{GaN}$ as substrates is that it can generate an inversion layer to accumulate electrons near the $\mathrm{SiO}_{2} / \mathrm{p}-\mathrm{GaN}$ interface. The accumulated electrons can easily recombine with the holes tunneling from graphene layer and create light emission. Notably, in order to generate the accumulation of electrons near the silicon oxide and semiconductor interface, the relative work functions between semiconductor and grapheme is very crucial. This criterion is very important for the extension of our current work to other material systems. Moreover, the resistivity of a compensated $\mathrm{GaN}$ film is much higher than that of doped $\mathrm{GaN}$, which is not suitable for the deployment in our light emitting device. Certainly, the LEM devices developed here can be extended to many other materials, including III-V or II-VI semiconductors, as long as the band alignment and work functions between the consituent elements fulfill the requirement for producing light emission.

Furthermore, in contrast to other frequently used conducting materials, graphene has been considered as a promising conducting material offering low cost and high transparency ${ }^{16}$. Notably, the $\mathrm{SiO}_{2}$ based memory structure has been widely used in RRAM cells due to its insulating property and has been well developed in recent years ${ }^{5-8}$. Because of the uniqueness of the LEM device shown here, our work could pave a milestone for the development of integrated optoelectronic devices and open up a new route for the application of optical communication, digital memories and recordable display panels.

\section{Results}

The graphene was synthesized by chemical vapor deposition (CVD) as described previously ${ }^{17}$. According to published reports, the absorption of graphene is strongly correlated to the number of graphene layers ${ }^{18}$. Therefore, we characterized the graphene layer by transmission spectrum, Raman spectrum as well as atomic force microscope (AFM). The obtained thickness and quality of the transferred graphene is evaluated from Raman spectrum, as shown in Figure 1a. The intensity ratio between $2 \mathrm{D}$ and $\mathrm{G}$ bands clearly reveals that our prepared graphene was single layer or bilayer. The AFM image shown here is to give an overview of the surface morphology after the deposition of graphene onto the underlying $\mathrm{p}-\mathrm{GaN}$ substrate, as shown in the inset of Figure 1a. Figure 1b shows the transmittance of the graphene in the wavelength range from $400 \mathrm{~nm}$ to $1100 \mathrm{~nm}$. It is clear that the graphene can be used as a substitute for transparent electrodes. Moreover, to measure the mobility of graphene and show how the quality of graphene is, a field-effect device
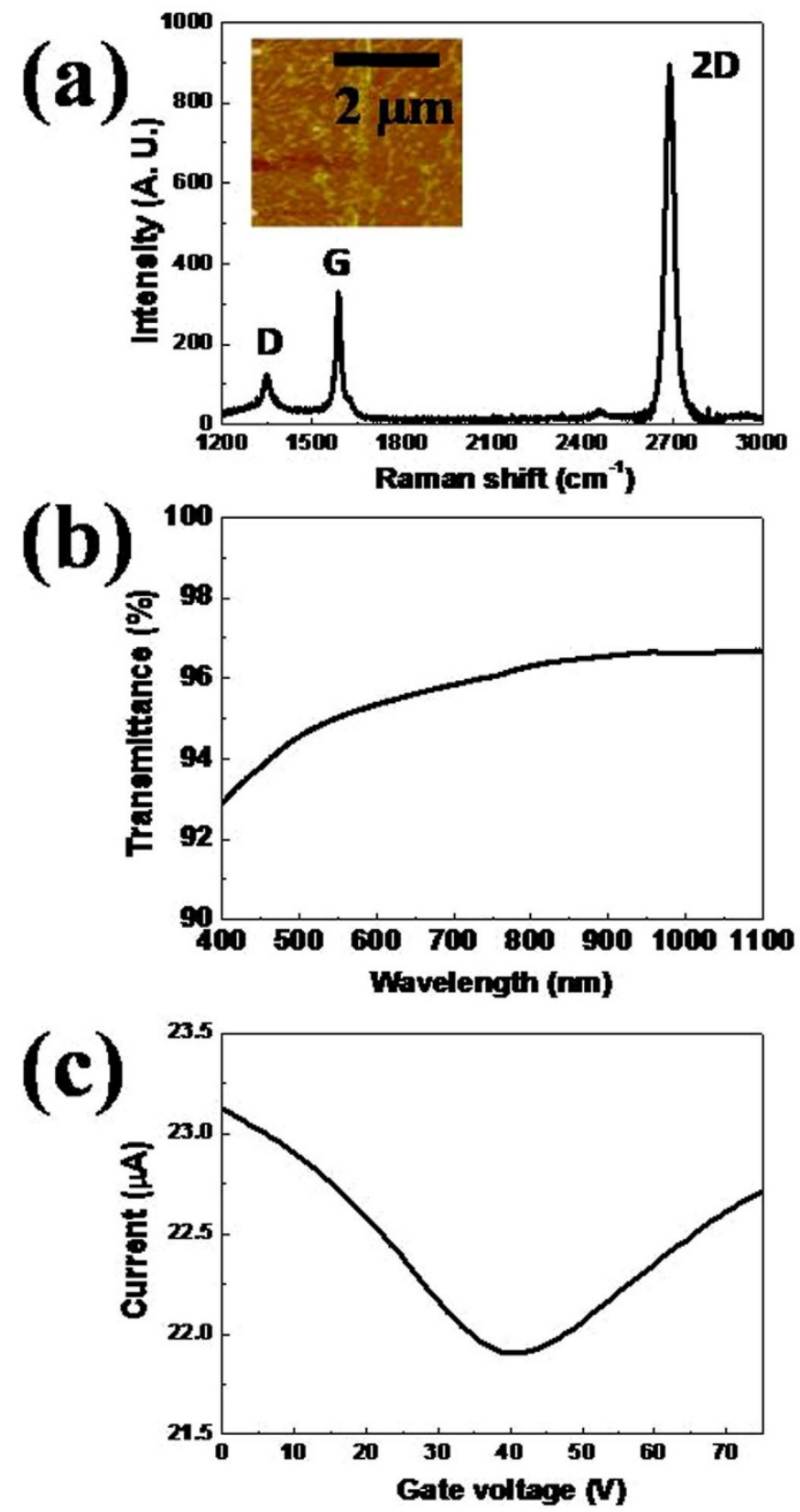

Figure $1 \mid$ (a) Raman spectrum and atomic force microscope (AFM) image (inset) of transferred graphene layer on $\mathrm{SiO}_{2}$. (b) Optical transmission spectrum of graphene. (c) Back-gate transfer characteristics of the graphene field-effect device.

made with graphene $/ \mathrm{SiO}_{2} / \mathrm{p}$-Si was constructed by a back-gate structure. Based on the field effect data, we are able to characterize the quality of the graphene layer and to obtain the carrier mobility. Figure 1c shows the back-gate transfer characteristics with a low source-drain voltage $(10 \mathrm{mV})$. The field-effect carrier mobility can be extracted from the linear regime as follows ${ }^{19}$

$$
\mu=\frac{L g_{m}}{W C_{o x} V_{d s}}
$$

where $\mathrm{L}$ and $\mathrm{W}$ are the channel length and the channel width, respectively, $g_{m}$ is the transconductance, $C_{o x}$ is the oxide capacitance and $\mathrm{V}_{\mathrm{ds}}$ is the channel bias. It is found that the field-effect carrier mobility of the graphene is about $1200 \mathrm{~cm}^{2} \mathrm{v}^{-1} \mathrm{~s}^{-1}$.

The configuration of the LEM is demonstrated in Figure 2a. The $\mathrm{SiO}_{2}$ layer was deposited by a radio-frequency sputtering system. The 
large-area CVD graphene was used as current spreading electrode, the $\mathrm{Ni} / \mathrm{Au}$ contact was used as current collecting electrode (cathode) and the Ag contact was used as control electrode (anode). In addition to serve as the transparent conducting layer, graphene also works as a stable interlayer without affecting the redox reaction in the formation of metal filament networks. Moreover, because the tunneling $\mathrm{SiO}_{2}$ layer only has a thickness of about $3 \mathrm{~nm}$, the transferred graphene on top of the $\mathrm{SiO}_{2}$ layer does not need the addition process for coating a metal film. It can avoid the damage of the thin $\mathrm{SiO}_{2}$ layer and prevent leakage current. In our device geometry, the top Ag electrode works as a reflective layer and the emitted light was detected from the p$\mathrm{GaN}$ side. If the graphene layer were replaced by coating other metal films with high reflectivity, it may affect the redox reaction in the formation of metal filament networks of the memory cell and induce leakage current. The inset of Figure 2 a shows the photograph of the LEM. Figure $2 b$ shows the photoluminescence (PL) spectrum of $\mathrm{p}$ $\mathrm{GaN}$ under light excitation of $325 \mathrm{~nm}$ at room temperature. The PL spectrum is dominated by a blue emission peak at $\sim 415 \mathrm{~nm}$ and a broadband yellow emission at $\sim 550 \mathrm{~nm}$. The emission peak at $\sim 415 \mathrm{~nm}$ could be mainly attributed to the energy level below the conduction band at $170 \mathrm{meV}$ (due to the interstitial $\mathrm{Mg}$ atoms) to the energy level above the valence band at $250 \mathrm{meV}$ (due to the acceptor level ${ }^{20}$. These doping-related energy levels strongly depend on the carrier density, that is, increase of the doping concentration results in the dominance of blue emission in the PL spectra ${ }^{21}$. Moreover, the annealing process for ohmic contact formation on $\mathrm{p}-\mathrm{GaN}$ can also induce red shift in PL spectrum ${ }^{22,23}$. Our result obtained here is consistent with previous reports that for moderated Mg-doped $\mathrm{GaN}$ with the hole concentration approaching $10^{17} \mathrm{~cm}^{-3}$, the PL spectra are dominated by the blue emission.

Bistable switching performance of the memory cell is important to control the luminescence arising from of the LEM. Therefore, we investigated the performance of the memory cell first. Figure $3 \mathrm{a}$ shows the $\mathrm{I}-\mathrm{V}$ characteristics of the $\mathrm{Ag} / \mathrm{SiO}_{2} /$ graphene memory cell at room temperature and the inset of Figure 3 a shows the schematics
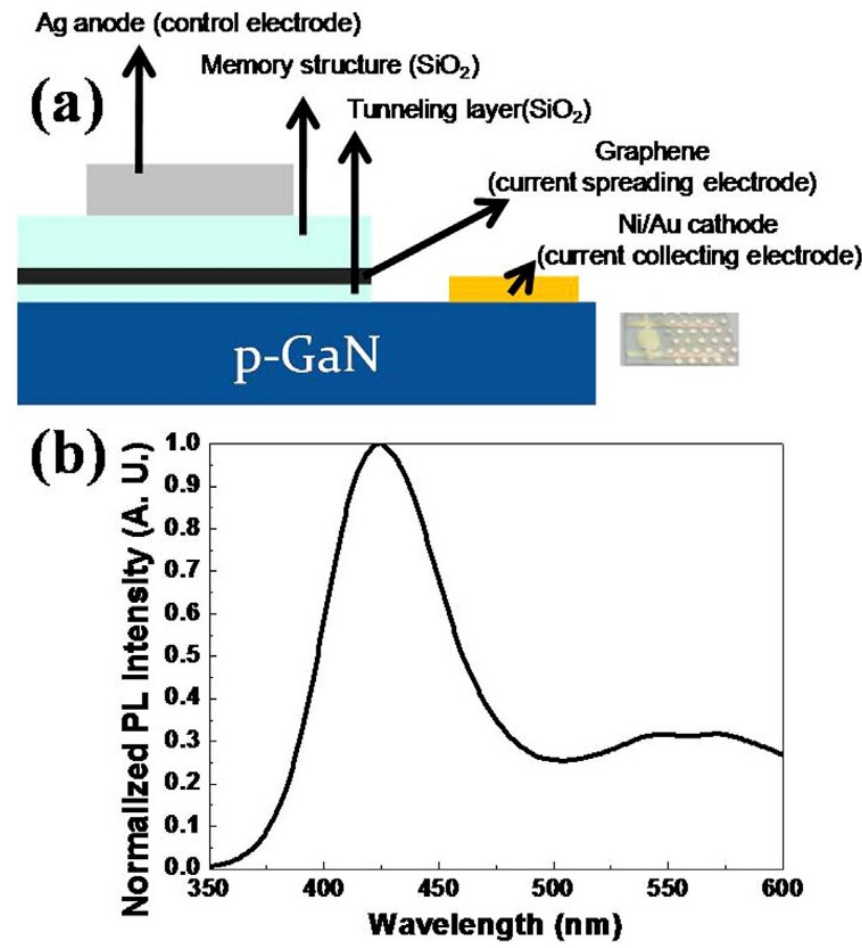

Figure $2 \mid$ (a) Schematics of light-emitting memory (LEM). The inset shows the photograph of the LEM. (b) Photoluminescence (PL) spectrum of $\mathrm{p}-\mathrm{GaN}$ at room temperature. view. As can be seen, the $\mathrm{Ag} / \mathrm{SiO}_{2} /$ graphene memory cell was at the HRS initially and showed a low-current characteristics in the lowvoltage range. When the applied voltage exceeded to a certain value, $\sim 3 \mathrm{~V}$ in Figure 3a, the injection current increased dramatically followed by an abrupt increase in the current flow and was switched from the HRS to the LRS, where the ON/OFF current ratio is about $10^{3}$. To prevent damage during the I-V measurements, we set a compliance current at $3 \mathrm{~mA}$. The state transition is equivalent to the "writing" command in the digital storage devices and the ON/ OFF current ratio promises a misreading probability in data access. According to previous reports, the conducting filament model with an electrochemical reaction is responsible for the resistive switching behavior $^{3-8}$. Hence, the state of $\mathrm{Ag} / \mathrm{SiO}_{2} /$ graphene memory cells can be switched between the HRS and the LRS using dc voltages. Switching perfromances between HRS and LRS were evaluated by performing the operation 100 times, as shown in Figure $3 \mathrm{~b}$. The current fluctuation at the high resistance state (HRS) may come from the incomplete dissolution of metal filament network ${ }^{24}$. It is clear that there was a little fluctuation of the HRS and LRS current levels and the ON/OFF ratio was quite stable. It is true that the on/off ratio is limited by the current compliance and is not representative of the device. The setting of the current compliance is used to prevent the device from damage. Indeed, a large on/off current ratio of more than $10^{4}$ can be obtained in our device. However, under such a high current operation, the reproducibility of the I-V curve becomes worse than the operation under the compliance current of $3 \mathrm{~mA}$.

Figure $4 \mathrm{a}$ shows the I-V characteristic of the LEM at room temperature, which is similar to the I-V characteristics shown in Figure 3a. The current flow sharply increased by 2 orders of magnitude at a critical voltage $(\sim 8 \mathrm{~V})$ and reflected the fact that the LEM
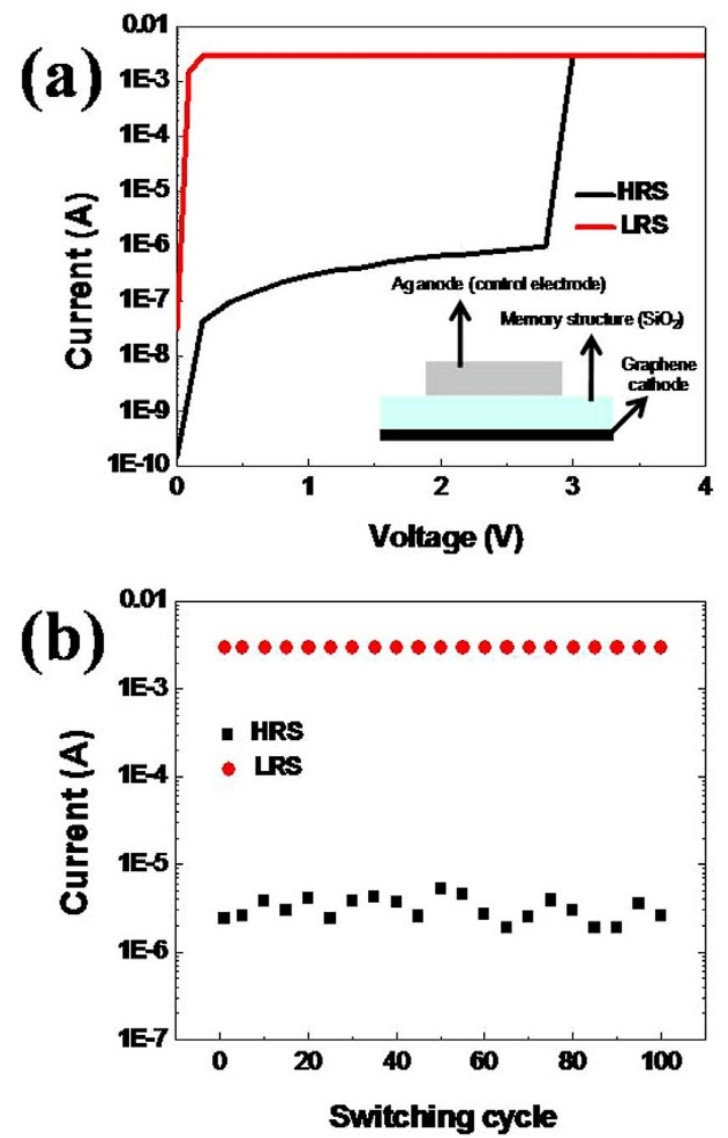

Figure 3 (a) I-V characteristics of $\mathrm{Ag} / \mathrm{SiO}_{2} /$ graphene memory cell. The inset shows the schematics of $\mathrm{Ag} / \mathrm{SiO}_{2} /$ graphene memory cell. (b) Switching behavior of $\mathrm{Ag} / \mathrm{SiO}_{2} /$ graphene memory cell over 100 cycles. 
was switched from the HRS to the LRS. The bistable switching performance of LEM is due to the bistability of $\mathrm{Ag} / \mathrm{SiO}_{2} /$ graphene memory cell. Noting that the LEM was achieved by developing a tandem structure, in which the writing voltage was expended since the voltage was applied to the graphene/SiO$/ 2 / p-G a N$ GIS-LED and the $\mathrm{Ag} /$ $\mathrm{SiO}_{2}$ /graphene memory cell. Because the I-V characteristics depend on the corresponding HRS or LRS, it is expected that the electroluminescence (EL) intensity of LEM should be different when the memory cell was switched from HRS to LRS. When the LEM is at the HRS, there is no EL signal until $\sim 8 \mathrm{~V}$ bias. However, when the LEM is at the LRS, the EL signal is detectable when the bias exceeds $\sim 6 \mathrm{~V}$. The emission state of LEM can be switched between the HRS and the LRS based on the I-V characteristics of the $\mathrm{Ag} / \mathrm{SiO}_{2} /$ graphene memory cell, as shown in the inset of Figure 4a. Switching perfromances between the HRS and LRS were evaluated by performing the operation 100 times, as shown in Figure $4 \mathrm{~b}$. It is clear that the switching characteristics of EL signal is also quite stable.

Figure $5 \mathrm{a}$ shows the EL spectra centered at $\sim 400 \mathrm{~nm}$ with different injection currents at room temperature. The emission peak is similar to that of the PL spectrum shown in Figure $2 \mathrm{~b}$. In order to clearly illustrate the underlying mechanism of the EL spectra, let us examine the band diagram of the graphene $/ \mathrm{SiO}_{2} / \mathrm{p}-\mathrm{GaN}$ GIS-LED in the LEM. Figure $5 b$ shows the band diagram of graphene $/ \mathrm{SiO}_{2} / \mathrm{p}$ GaN GIS-LED at thermal equilibrium according to the data reported previously ${ }^{25,26}$. Since the $\mathrm{SiO}_{2}$ layer acts as an energy barrier, the carrier injected from graphene responsible for the radiative recombination of the light emission must tunnel through the $\mathrm{SiO}_{2}$ layer,
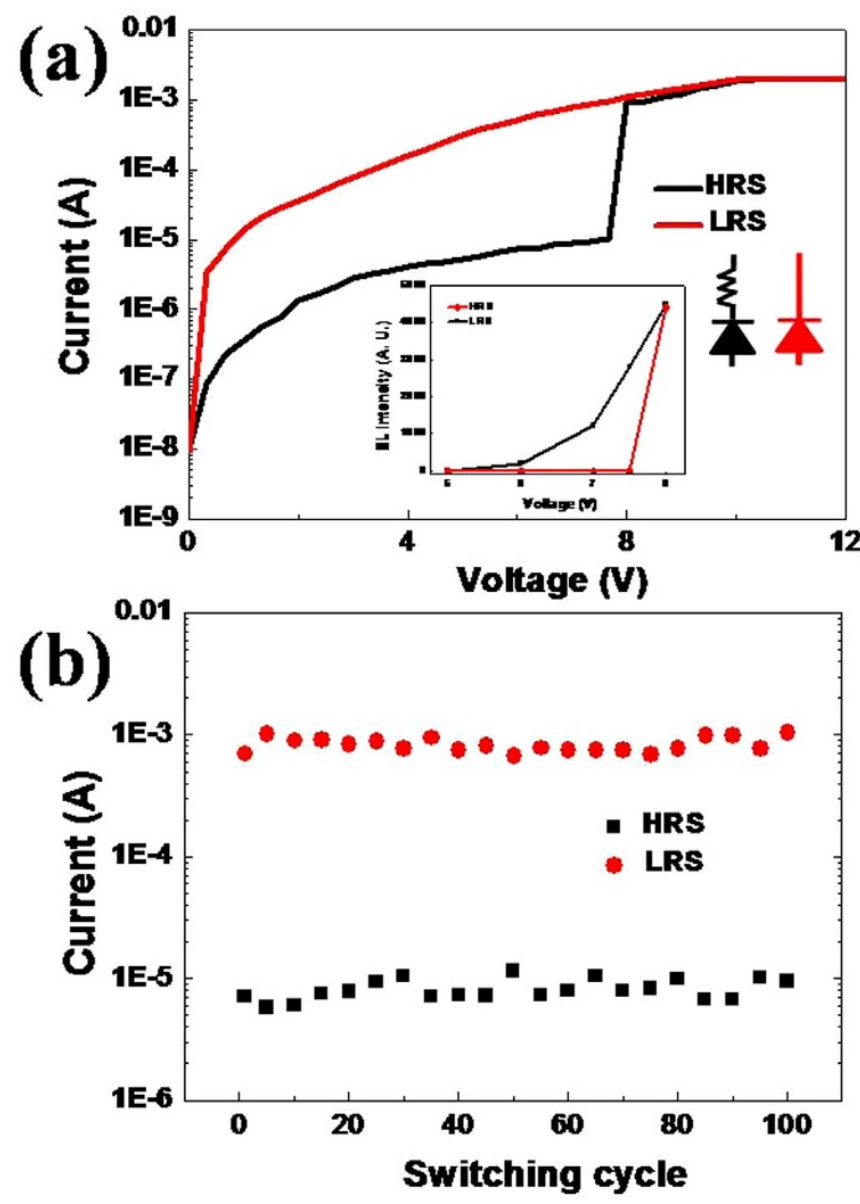

Figure 4 | (a) I-V characteristics of light-emitting memory (LEM). The inset shows electroluminescence (EL) intensity of LEM as a function of voltage at high resistance state (HRS) and low resistance state (LRS). (b) Switching behavior of LEM over 100 cycles. which is quite different from that of the common $p-n$ junctions. When applying a positive bias on graphene, most of the voltage drops across the $\mathrm{SiO}_{2}$ layer because graphene and $\mathrm{p}-\mathrm{GaN}$ have relatively low resistance in comparison with $\mathrm{SiO}_{2}$, as shown in Figure $5 c$. The applied electric-field will induce the band bending of $\mathrm{GaN}$ at the $\mathrm{SiO}_{2} / \mathrm{p}-\mathrm{GaN}$ interface and introduce an inversion layer with the accumulated electrons. Meanwhile, the Fermi level of graphene moves downward, which enables the holes in graphene to tunnel into the valence band of $\mathrm{p}-\mathrm{GaN}$ and cause the EL signal. The origin of UV emission can be attributed to the recombination of the trapped holes in deep acceptor levels with electrons in the conduction band.

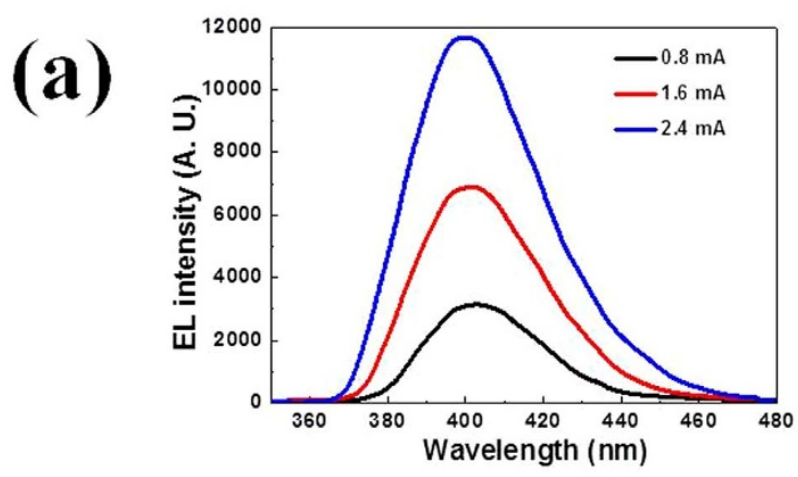

Thermal equilibrium
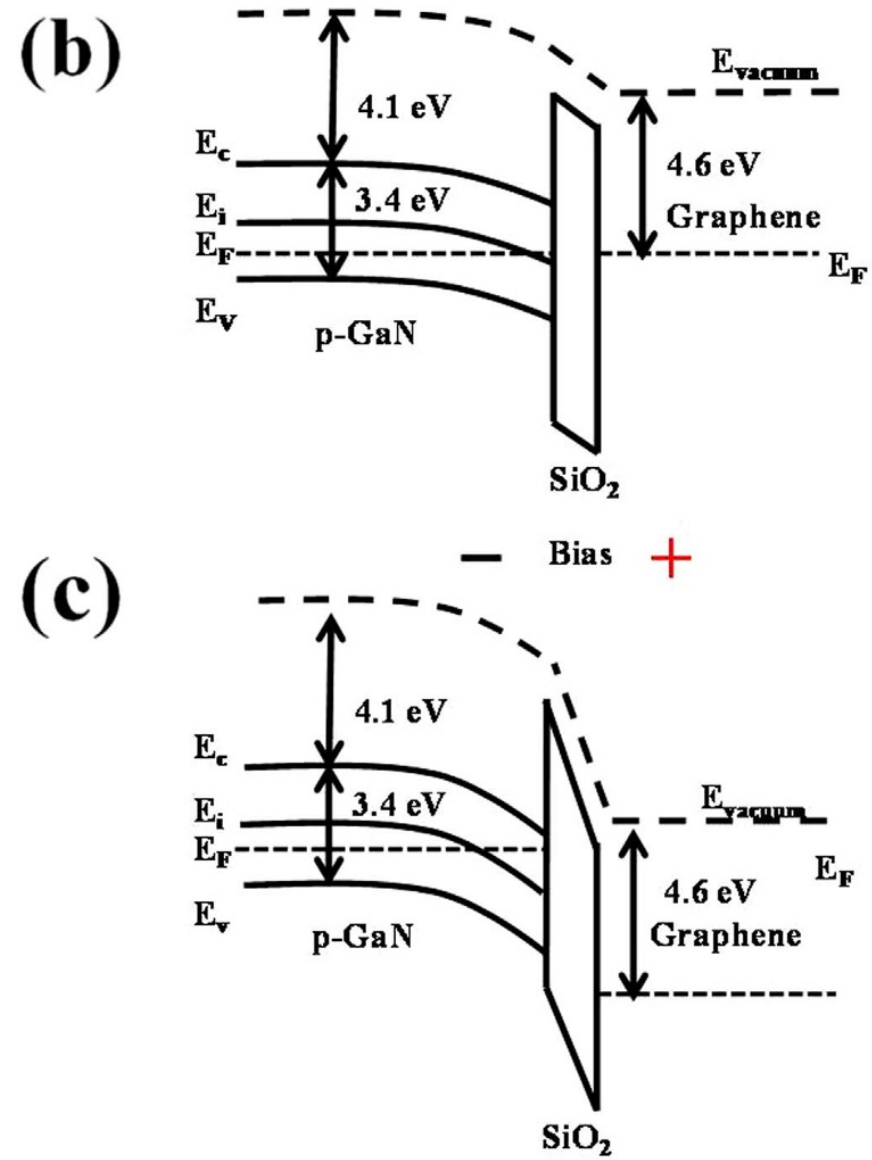

Figure $5 \mid$ (a) Electroluminescence (EL) spectra of light-emitting memory (LEM) with different injection current. (b) Energy band diagram of graphene/ $\mathrm{SiO}_{2} / \mathrm{p}-\mathrm{GaN}$ metal/insulator/semiconductor light-emitting diode (GIS-LED) under thermal equilibrium. (c) Energy band diagram of graphene $/ \mathrm{SiO}_{2} / \mathrm{p}-\mathrm{GaN}$ GIS-LED under bias. 


\section{Discussion}

To further understand the conduction mechanisms of LEM, we qualitatively analyze the relationship between the injected current I and the applied bias $\mathrm{V}$ of the $\mathrm{Ag} / \mathrm{SiO}_{2} /$ graphene memory cell first. Figure 6 shows the logarithmic plot and linear fitting of HRS curve in Figure 3a. The I-V curve of HRS exhibits a linear relationship between injection current and bias with a slope of 1.15 , which is very close to 1 and matches the Ohmic conduction ${ }^{27}$. The Ohmic conduction can be expressed as,

$$
I \propto V \exp \left(\frac{-E_{a}}{k T}\right),
$$

where $\mathrm{E}_{\mathrm{a}}$ is the activation energy of electron. At the LRS, the injection current increased dramatically and saturated, which is dominated by the conducting filament model, as described in previous reports ${ }^{3-8}$.

Similarly to the case of $\mathrm{Ag} / \mathrm{SiO}_{2} /$ graphene memory cell, we can deduce the conduction mechanisms of LEM from the typical plots of the I-V characteristics. As shown in Figure 7, the conduction mechanism of LEM is much more complicated and each HRS and LRS I-V curve can be divided into two portions. From $0 \mathrm{~V}$ to $7 \mathrm{~V}$ at the HRS, the LEM exhibits a logarithmic relationship between injection current and bias with a slope of 1.4. It is a consequence of the combination of the I- $\mathrm{V}$ characteristics of $\mathrm{Ag} / \mathrm{SiO}_{2} /$ graphene memory cell and graphene $/ \mathrm{SiO}_{2} / \mathrm{p}$-GaN GIS-LED, but the $\mathrm{Ag} / \mathrm{SiO}_{2} /$ graphene memory cell dominates the conduction mechanism at HRS. When bias is above $8 \mathrm{~V}$ at the HRS, an abrupt increase in current occurs, and the I-V characteristic exhibits a typical Fowler-Nordheim (FN) tunneling curve with a negative slope as shown in Figure 7b. According to the previous reports ${ }^{28}$, within the Simmon's approximation, in the limit of a large applied bias, the FN tunneling can be written as,

$$
I \propto V^{2} \exp \left(-\frac{4 d \sqrt{2 m^{*} \phi^{3}}}{3 \hbar e V}\right),
$$

where $d$ is the barrier width, $m^{*}$ is the electron effective mass and $\phi$ is the tunnel barrier height. However, for the LEM at LRS, the I-V characteristics can be well fitted by direct tunneling and FN tunneling at low applied bias $(<4 \mathrm{~V})$ and high applied bias $(>4 \mathrm{~V})$, respectively. The tunneling relationship for direct tunneling can be expressed by the following equations ${ }^{28}$

$$
I \propto V \exp \left(-\frac{2 d \sqrt{2 m^{*} \phi}}{\hbar}\right),
$$

The fitting I-V characteristics of LEM at LRS is shown in Figure 7c, which is in good agreement with the theoretical prediction. Linear decrease at low bias region and linear increase at high bias voltage

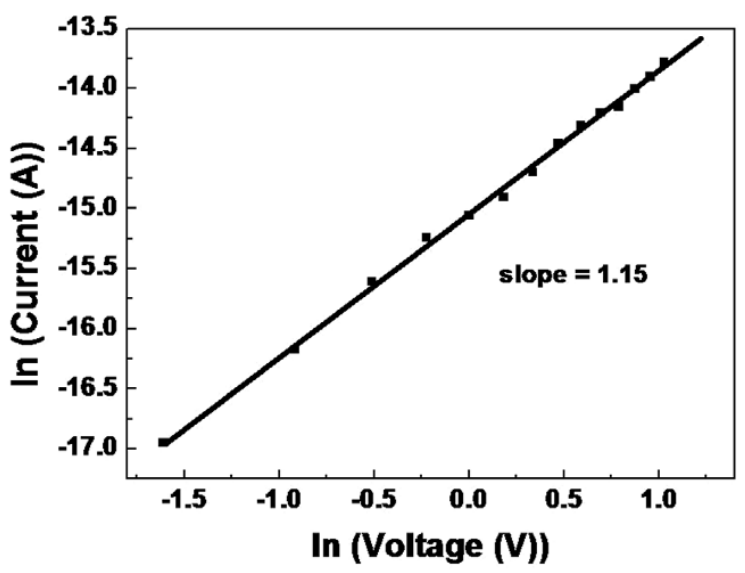

Figure 6 The logarithmic plot and linear fitting of $\mathrm{Ag} / \mathrm{SiO}_{2} /$ graphene memory cell at high resistance state (HRS).
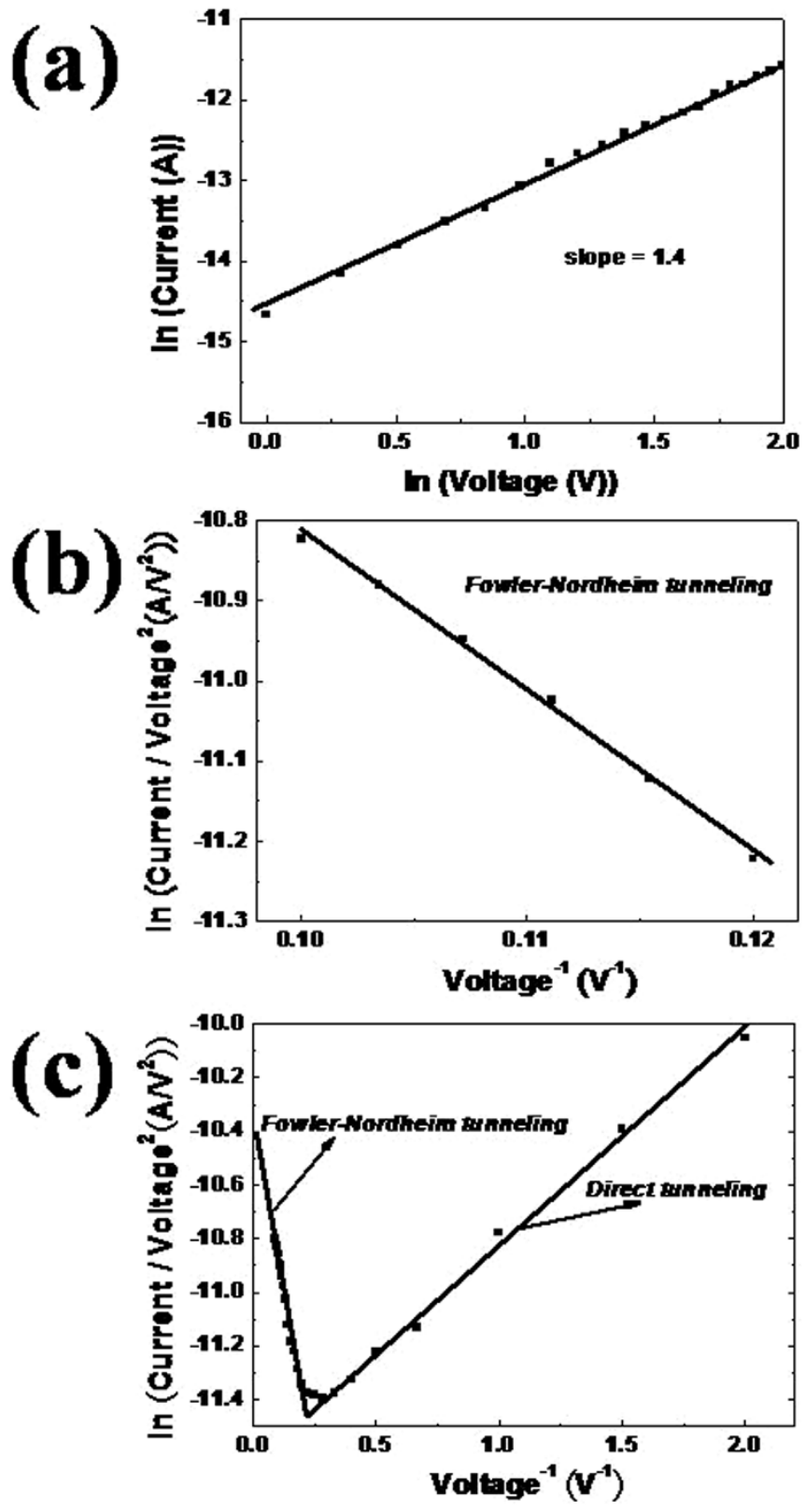

Figure $7 \mid$ (a) The logarithmic plot and linear fitting of light-emitting memory (LEM) at high resistance state (HRS) before $8 \mathrm{~V}$ bias. (b) The current intensity of LEM, divided by $\mathrm{V}^{2}$, as a function of $\mathrm{V}^{-1}$ at HRS after $8 \mathrm{~V}$ bias. (c) The current intensity of LEM, divided by $\mathrm{V}^{2}$, as a function of $\mathrm{V}^{-1}$ at low resistance state (LRS).

indicate that the conduction mechanisms in LEM at LRS are based on direct tunneling and FN tunneling at low applied biases and high applied biases, respectively. This result is reasonable because FN tunneling is a conduction mechanism, where the carriers tunneling through a triangular barrier requires a high electric-field. The above analysis of the conduction mechanism provides a firm evidence to support the fact that the tunneling process plays a very important role for the EL in our LEM devices.

Finally, let us provide a discussion for the optimization of the on/ off current ratio and the reduction of the threshould voltage for the LEM device. The thickness of the $\mathrm{SiO}_{2}$ layer above the graphene layer is $50 \mathrm{~nm}$ and is about $3 \mathrm{~nm}$ below the graphene layer. Clearly, the threshold voltage and the HRS current will increase and decrease with respect to the increase of the thickness of the upper and lower $\mathrm{SiO}_{2}$ layers, respectively. For instance, in the LHS, the current is 
dominated by the tunneling through the lower $\mathrm{SiO}_{2}$ layer. As shown in equations (3) and (4), the relationship between the tunneling current and the $\mathrm{SiO}_{2}$ thickness follows an exponential function. A small increase in the $\mathrm{SiO}_{2}$ thickness can drastically reduce the magnitude of the tunneling current. According to the above description, the key factor to have a higher ON/OFF ratio and lower threshold voltage for our LEM device does strongly depend on the thickness of the upper and lower $\mathrm{SiO}_{2}$ layers. The decrease of the thickness of the lower $\mathrm{SiO}_{2}$ layer will result in a lower threshold voltage and a higher current in the LRS. Consequently, a smaller thickness of the lower $\mathrm{SiO}_{2}$ layer without leakage current is the best choice. On the other hand, the decrease of the thickness of the upper $\mathrm{SiO}_{2}$ layer will increase the current in the HRS and decrease the threshold voltage. Therefore, the thickness of the upper $\mathrm{SiO}_{2}$ layer has to be optimized in order to have a higher on/off ratio and a lower threshold voltage. Another excellent alternative to have a higher on/off ratio and lower threshold voltage is to replace the GIS-LED developed here by the well mature and commercially available III-V compound LEDs.

In summary, we have developed a new LEM with bistable luminescence control. The success of LEM mainly arises from the integration of light emitters and memories with a graphene interlayer, which combines several advantages of the composed materials. The LEM was achieved by developing the GIS-LED in tandem with a $\mathrm{SiO}_{2}$ based memory cell. The underlying mechanism of the observed EL spectra can be interpreted quite well by the carrier tunneling through the insulating layer, which is very different from the standard p-n junction model. The feasibility of the new GIS-LED is made possible by the excellent properties of conductivity and transparency possessed by graphene. It is emphasized that the new LEM enjoys a simple structure and can be fabricated by using mature facilities. It is ready to be extended into many other material systems for practical applications. In view of the unique features demonstrated by the integration of light emitters and memories, the approach shown here may open up a new route for the development of integrated optoelectronic devices and pave a milestone for optical communication, digital memories and recordable display panels.

\section{Methods}

We grew graphene on copper foils at temperature up to $1000^{\circ} \mathrm{C}$ by CVD process and the mixture of methane and hydrogen was used as carbon source. At the temperature around $1000^{\circ} \mathrm{C}$, the carbon atoms from gaseous reactants are deposited onto the metal substrate via chemical adsorption.Polymethyl-methacrylate (PMMA) was spun-coated on the graphene to transfer and align graphene on $\mathrm{p}-\mathrm{GaN}$.

The device structure of LEM consists of $\mathrm{Ag} / \mathrm{SiO}_{2} / \mathrm{graphene} / \mathrm{SiO}_{2} / \mathrm{p}-\mathrm{GaN}$. The $\mathrm{p}-$ $\mathrm{GaN}$ was doped by $\mathrm{Mg}$ with the hole concentration in the range of $6 \times 10^{16} \mathrm{~cm}^{-3}$. Initially, p-GaN wafer was rinsed in acetone, isopropanol and $\mathrm{DI}$ water. $\mathrm{Ni} / \mathrm{Au}$ was firstly deposited on $\mathrm{p}-\mathrm{GaN}$ with annealing to obtain a ohmic contact. The first $\mathrm{SiO}_{2}$ layer with a thickness of $3 \mathrm{~nm}$ was grown on $\mathrm{p}-\mathrm{GaN}$ via RF sputtering after the formation of $\mathrm{Ni} / \mathrm{Au}$ ohmic contact. The graphene layer was then assembled by a transfer process on $\mathrm{p}-\mathrm{GaN}$. The second $\mathrm{SiO}_{2}$ layer with a thickness of $50 \mathrm{~nm}$ was sequentially deposited on top of graphene. The LEM was completed by the deposition of Ag electrode to serve as anode.

1. Lundstrom, M. Moore's law forever? Science 299, 210-211 (2003).

2. Leong, M., Doris, B., Kedzierski, J., Rim, K. \& Yang, M. Silicon device scaling to the sub-10-nm regime. Science 306, 2057-2060 (2004).

3. Ma, L. P., Liu, J. \& Yang, Y. Organic electrical bistable devices and rewritable memory cells. Appl. Phys. Lett. 80, 2997 (2002).

4. Sawa, A. Resistive switching in transition metal oxides. Mater. Today 11, 28-36 (2008).

5. Waser, R., Dittmann, R., Staikov, G. \& Szot, K. Redox-based resistive switching memories-nanoionic mechanisms, prospects, and challenges. Adv. Mater. 21, 2632-3663 (2009)

6. Schindler, C., Staikov, G. \& Waser, R. Electrode kinetics of $\mathrm{Cu}-\mathrm{SiO} 2$-based resistive switching cells: Overcoming the voltage-time dilemma of electrochemical metallization memories. Appl. Phys. Lett. 94, 072109 (2009).
7. Yao, J., Sun, Z., Zhong, L., Natelson, D. \& Tour, J. M. Resistive switches and memories from silicon oxide. Nano Lett. 10, 4105-4110 (2010).

8. Yang, Y. et al. Observation of conducting filament growth in nanoscale resistive memories. Nat. Commun. 3, 732 (2012).

9. Nakamura, S., Senoh, M. \& Mukai, T. High-power InGaN/GaN doubleheterostructure violet light emitting diodes. Appl. Phys. Lett. 62, 2390 (1993).

10. Akasaki, I. et al. Shortest wavelength semiconductor laser diode. Electron. Lett. 32 , 1105-1106 (1996).

11. Waltereit, P. et al. Nitride semiconductors free of electrostatic fields for efficient white light-emitting diodes. Nature 406, 865-868 (2000).

12. Kim, H. M., Kang, T. W. \& Chung, K. S. Nanoscale ultraviolet-light-emitting diodes using wide-bandgap gallium nitride nanorods. Adv. Mater. 15, 567-569 (2003).

13. Wierer, J. J. et al. InGaN/GaN quantum-well heterostructure light-emitting diodes employing photonic crystal structures. Appl. Phys. Lett. 84, 3885 (2004).

14. Gardner, N. F. et al. Blue-emitting InGaN-GaN double-heterostructure lightemitting diodes reaching maximum quantum efficiency above $200 \mathrm{~A} / \mathrm{cm}^{2}$. Appl. Phys. Lett. 91, 243506 (2007).

15. Kwon, M. K. et al. Surface-plasmon-enhanced light-emitting diodes. Adv. Mater. 20, 1253-1257 (2008).

16. Li, X. et al. Large-area synthesis of high-quality and uniform graphene films on copper foils. Science 324, 1312-1314 (2009).

17. Tan, W. C., Hofmann, M., Hsieh, Y. P., Lu, M. L. \& Chen, Y. F. A graphene-based surface plasmon sensor. Nano Res. 5, 695-702 (2012).

18. Wang, Y., Tong, S. W., Xu, X. F., Özyilmaz, B. \& Loh, K. P. Interface engineering of layer-by-layer stacked graphene anodes for high-performance organic solar cells. Adv. Mater. 23, 1514 (2011).

19. Nayfeh, O. M. Radio-frequency transistors using chemical-vapor-deposited monolayer graphene: performance, doping, and transport effects. IEEE Trans. Electron Devices 58, 2847 (2011).

20. Smith, M. et al. Mechanisms of band-edge emission in Mg-doped p-type GaN. Appl. Phys. Lett. 68, 1883 (1996).

21. Guarneros, C. \& Sánchez, V. Magnesium doped GaN grown by MOCVD. Mater. Sci. Eng. B 174, 263 (2010).

22. Götz, W., Johnson, N. M., Walker, J., Bour, D. P. \& Street, R. A. Activation of acceptors in $\mathrm{Mg}$-doped $\mathrm{GaN}$ grown by metalorganic chemical vapor deposition. Appl. Phys. Lett. 68, 667 (1996).

23. Shahedipour, F. \& Wessels, B. W. Investigation of the formation of the $2.8 \mathrm{eV}$ luminescence band in p-type GaN: Mg. Appl. Phys. Lett. 76, 3011 (2000).

24. $\mathrm{Hu}, \mathrm{W}$. et al. Bipolar and tri-state unipolar resistive switching behaviors in $\mathrm{Ag} /$ ZnFe2O4/Pt memory devices. Appl. Phys. Lett. 101, 063501 (2012).

25. Weinberg, Z. A., Rubloff, G. W. \& Bassous, E. Transmission, photoconductivity, and the experimental band gap of thermally grown $\mathrm{SiO}_{2}$ films. Phys. Rev. B 19, 3107-3117 (1979).

26. Vurgaftman, I., Meyer, J. R. \& Ram-Mohan, L. R. Band parameters for III-V compound semiconductors and their alloys. J. Appl. Phys. 89, 5815 (2001).

27. Sze, S. M. Physics of Semiconductor Devices. (Wiley, 1981).

28. Simmons, J. G. Generalized formula for the electric tunnel effect between similar electrodes separated by a thin insulating film. J. Appl. Phys. 34, 1793 (1963).

\section{Acknowledgments}

This work was supported by the National Science Council and Ministry of Education of the Republic of China.

\section{Author contributions}

C.W.C. and Y.F.C. designed the concept. W.C.T. synthesized the material. M.L.L. and T.C.P. performed the experiments. C.W.C., Y.J.Y. and Y.F.C. discussed the results. C.W.C and Y.F.C. supervised the project and finalized the manuscript.

\section{Additional information}

Competing financial interests: The authors declare no competing financial interests.

How to cite this article: Chang, C.-W. et al. Electrically and Optically Readable Light Emitting Memories. Sci. Rep. 4, 5121; DOI:10.1038/srep05121 (2014).

This work is licensed under a Creative Commons Attribution-NonCommercialNoDerivs 3.0 Unported License. The images in this article are included in the article's Creative Commons license, unless indicated otherwise in the image credit; if the image is not included under the Creative Commons license, users will need to obtain permission from the license holder in order to reproduce the image. To view a copy of this license, visit http://creativecommons.org/licenses/by-nc-nd/3.0/ 